\title{
An Integral-Equation Theory for a Self-Interacting Polymer Adsorbed at an Interface
}

\author{
Jun Cai and John M. Prausnitz ${ }^{\text {a) }}$ \\ Department of Chemical Engineering, University of California, Berkeley, and Chemical Sciences Division, \\ Lawrence Berkeley National Laboratory, Berkeley, California 94720
}

\begin{abstract}
An integral-equation theory based on the Born-Green-Yvon (BGY) hierarchy for a self-interacting polymer is used to describe a polymer adsorbed at an oil-water interface. The polymer is represented by a square-well chain. The interaction between a polymer segment and an oil-water interface is represented by an asymmetric square-well potential where the well-depth on one side reflects water-polymer and the well depth on the other side reflects oil-polymer interactions. To truncate the BGY hierarchy, we introduce two approximations: first we use the Markov-chain approximation for intra-molecular correlation functions, and second, we use the effective intra-molecular energy in the bulk to approximate that at the interface. The results are compared with Monte-Carlo-simulation data. For short chains, when the attractive interaction between the segments is weak, the theory is in good agreement with Monte Carlo simulation. Stronger segment-segment attractive interactions increase adsorption.
\end{abstract}

\section{INTRODUCTION}

Adsorption of a polymer at a liquid-liquid interface plays an important role in stabilization of colloidal particles, in lubrication, in membrane phenomena and in numerous biological systems [1-6]. When a long, flexible polymer chain adsorbs at the oil-water interface, it is often adsorbed so strongly that, essentially, it remains at the interface indefinitely; this phenomenon, discussed by De Gennes over 20 years ago [1], has received wide attention [2-6]. To describe this adsorption, the system is usually modeled as a polymer in an external field due to the wall or interface [7-9].

Several theories in polymer physics can be used to explain adsorption of the polymer at an interface; the most important theory is Gaussian-chain theory that leads to a diffusion or Schrödinger-like equation [10]. For the simplest case (no interaction between segments other than bond energy), we obtain the general solution of the diffusion equation [2] that is a function of

a) Author to whom correspondence should be addressed. Email: prausnit@cchem.berkeley.edu 
eigenvalues. For the more general case, perturbation methods or numerical methods must be used to obtain an asymptotic solution [7-9, 11] or numerical results [12]. Gaussian-chain theory provides not only a qualitative description of the pertinent polymer physics, but also provides a basis for modern polymer theories.

When the segment-segment interaction cannot be neglected, we require a more sophisticated theory, such as self-consistent field (SCF) theory, perturbation theory and renormalization-group (RG) theory. Self-consistent field (SCF) theory in polymer physics, pioneered by Edwards [13] and developed by Freed et al [14-16], is used to solve the polymer excluded-volume problem [17]. In SCF theory, excluded-volume problems are transformed into a description of a Gaussian chain in an effective external field due to repulsive self-interactions. This effective field takes into account self-consistently the effect of excluded volume. In addition to excluded-volume problems, SCF is also used to describe the coil-globule transition where the segment-segment interaction changes from repulsive to attractive [18]. To describe scaling behavior, $\mathrm{RG}$ theory [3, 7-8, 10, 19-21], combined with either a perturbation method or SCF, is needed to take into account long-range correlations due to connections between segments.

In field theory, because the polymer is very long, details of interactions between segments are not important. However, in our previous work [22], we found that such a field description is not sufficient to describe polymers with attractive interactions between segments adsorbed at the interface. We found a bound state for a polymer even when the external field vanishes. This bound state is used to explain the collapse of a chain in the bulk. But for an inhomogeneous system, this bound state is physically meaningless. This problem probably arises from the using of the Dirac function to represents the segment-segment interaction. In such a description of interactions between segments, because the attractive interaction is pair-wise, the repulsive interaction has to be ternary. However, the repulsive interactions and attractive interactions should have an equal status; both should be either binary or ternary.

For a more realistic segment-segment potential model, (e.g. a square-well chain or a Lennard-Jones chain), the integral-equation theory of liquids is more suitable [23-28]. An advantage of integral-equation theory is that its results can be compared with Monte Carlo simulations. Based on a new version of the Kirkwood superposition approximation, Eu and Gan (EG) $[25,26]$ derived a hierarchy of intra-molecular correlation functions for molecular liquids. Another well-known integral equation for a single polymer chain is the Born-Green-Yvon (BGY) equation that appeared several decades ago [29, 30]. Both EG and BGY theories are used to 
describe the polymer in the bulk. They predicted the scaling law for end-to-end distance when the temperature is not very low.

In this work, we develop an integral-equation theory for a polymer adsorbed at the interface based on the BGY hierarchy. We use the first equation of the BGY hierarchy to calculate the density distribution of a single chain adsorbed at an oil-water interface that is modeled by an external potential. To truncate the BGY hierarchy, we introduce two approximations. The first approximation is a Markov-chain-like approximation and the second one concerns the calculation of the end-to-end correlation function of a real chain at the interface. Our results are compared with Monte-Carlo-simulation data.

\section{THEORY}

The polymer is modeled as a freely jointed chain whose segments are hard spheres with short-range attraction between non-bonded segments. The solvent is a continuous medium; interactions between solvent and polymer are integrated into the effective interaction between segments. For the interface energy, we use our previous model [31]: the interface is modeled by an asymmetric square-well potential where oil and water are continuous media. The oil-water interface is in the $x-y$ plane; coordinate $z$ is perpendicular to that plane. Because we use the Green function of a Gaussian chain in this work and because the external potential of a Gaussian chain is given by a per-unit-chain length not by per segment, for simplicity and to make the external potential of a hard chain consistent with that of a Gaussian chain, we assume that the external potential of two end segments of a hard or square-well chain is half that of other segments. Such a definition will not significantly influence the results. The external potential per segment (not the end segment) is given by:

$$
\beta v(z)=\left\{\begin{array}{c}
+\infty ; \quad z<-H_{1} \text { or } z>H_{2} \\
\beta\left(\varepsilon_{1}-\varepsilon_{0}\right) ; \quad-H_{1} \leq z \leq-a / 2 \\
-\beta \varepsilon_{0} ; \quad-a / 2<z<a / 2 \\
0 ; \quad a / 2 \leq z \leq H_{2}
\end{array}\right.
$$

where $\varepsilon_{0}, \varepsilon_{1} \geq 0$ are the well depths, one on the water side and the other on the oil side; $a$ is the well width and $H_{1}+H_{2}$ is the width of the system in the $z$ direction; and $\beta=(k T)^{-1}$. For a Gaussian chain, it was shown [9] that there exists a critical value of chain length depending on the external potential. The polymer adsorbs at the interface if its length is larger than this critical value; 
otherwise the polymer is distributed over the whole space, i.e. the density is zero everywhere if the space is infinitely large. Our previous work [31] also showed that larger values of $H_{1}$ and $H_{2}$ do not affect the density distribution close to the limiting distribution but reduce the density distribution for a short chain. Although these results are for a Gaussian chain, we expect that they also hold for a real chain. Thus in this work, to simplify the problem and to ensure that the density distribution is not zero, we add two walls on the $z$-axis.

The canonical partition function of a single chain containing $N$ segments is given by:

$$
Z_{N}=V^{-1}\left(H_{1}+H_{2}\right) \int \cdots \int \prod_{i=1}^{N-2} \prod_{j=i+2}^{N} \exp \left(-\beta \phi_{i j}\right) \prod_{i=1}^{N} \exp \left(-\beta v_{i}\right) \prod_{i=1}^{N-1} s_{i, i+1} \prod_{i=1}^{N} \mathrm{~d} \mathbf{r}_{i}
$$

where $V$ is the volume of the system; subscripts $i, j$ are the ordinal numbers of the segments; $\phi_{i j}$ is the non-bonding interaction between segments $i$ and $j ; s_{i, i+1}$ is the Boltzmann factor for the bonding energy between segments $i$ and $i+1 ; v_{i}$ is the external potential of segment $i$. According to Eq. (1), $v_{1}=v_{N}=v / 2$ and $v_{2}=\cdots=v_{N-1}=v$. The factor $V^{-1}\left(H_{1}+H_{2}\right)$ is added to make the partition function consistent with the normalization condition given by Eq. (5) and Eq. (9). The bonding energy is given by:

$$
s_{i, i+1}\left(\mathbf{r}_{i}, \mathbf{r}_{i+1}\right)=\frac{1}{4 \pi \sigma^{2}} \delta\left(\left|\mathbf{r}_{i}-\mathbf{r}_{i+1}\right|-\sigma\right)
$$

where $\sigma$ is the diameter of the hardcore of the segment. The density distribution function of segment $k$ is defined by:

$$
\rho_{k}\left(\mathbf{r}_{k}\right)=Z_{N}^{-1} \int \cdots \int \prod_{i=1}^{N-2} \prod_{j=i+2}^{N} \exp \left(-\beta \phi_{i j}\right) \prod_{i=1}^{N} \exp \left(-\beta v_{i}\right) \prod_{i=1}^{N-1} s_{i, i+1} \prod_{i \neq k}^{N} \mathrm{~d} \mathbf{r}_{i} .
$$

According to the symmetry of the external potential, Eq. (1), the density distribution is only concerned with the coordinate $z_{k}$. Thus, the density distribution function satisfies the normalization condition given by:

$$
\int \rho_{k}(z) \mathrm{d} z=1 .
$$

Calculating the derivative of the distribution function in Eq. (4) with respect to $z$, we obtain

$$
\begin{aligned}
& \frac{\mathrm{d} \rho_{k}\left(z_{k}\right)}{\mathrm{d} z_{k}}=\rho_{k}\left(z_{k}\right) \frac{\mathrm{d}\left[-\beta v_{k}\left(z_{k}\right)\right]}{\mathrm{d} z_{k}}+\sum_{j \neq k, k \pm 1}^{N} \int \mathrm{d} \mathbf{r}_{j} w_{k j}\left(\mathbf{r}_{k}, \mathbf{r}_{j}\right) \frac{\partial\left[-\beta \phi_{k j}\left(\mathbf{r}_{k}, \mathbf{r}_{j}\right)\right]}{\partial z_{k}} \\
& +\int \mathrm{d} \mathbf{r}_{k-1} w_{k-1, k}\left(\mathbf{r}_{k-1}, \mathbf{r}_{k}\right) \frac{\partial \ln s_{k-1, k}\left(\mathbf{r}_{k-1}, \mathbf{r}_{k}\right)}{\partial z_{k}}+\int \mathrm{d} \mathbf{r}_{k+1} w_{k, k+1}\left(\mathbf{r}_{k}, \mathbf{r}_{k+1}\right) \frac{\partial \ln s_{k, k+1}\left(\mathbf{r}_{k}, \mathbf{r}_{k+1}\right)}{\partial z_{k}}
\end{aligned}
$$


where $w_{k j}\left(\mathbf{r}_{k}, \mathbf{r}_{j}\right)$ is the intra-molecular correlation function between segments $i$ and $j$ defined by:

$$
w_{k j}\left(\mathbf{r}_{k}, \mathbf{r}_{j}\right)=Z_{N}^{-1} \int \cdots \int \prod_{i=1}^{N-2} \prod_{j=i+2}^{N} \exp \left(-\beta \phi_{i j}\right) \prod_{i=1}^{N} \exp \left(-\beta v_{i}\right) \prod_{i=1}^{N-1} s_{i, i+1} \prod_{i \neq k, j}^{N} \mathrm{~d} \mathbf{r}_{i}
$$

Because of the symmetry of the external potential, we have

$$
w_{k j}\left(\mathbf{r}_{k}, \mathbf{r}_{j}\right)=w_{k j}\left(z_{k}, z_{j}, R_{k j}\right)
$$

where $R_{k j}=\sqrt{\left(x_{k}-x_{j}\right)^{2}+\left(y_{k}-y_{j}\right)^{2}}$. The intra-molecular correlation function satisfies the normalization condition:

$$
\int_{-H_{2}}^{H_{1}} \mathrm{~d} z_{k} \int_{-H_{2}}^{H_{1}} \mathrm{~d} z_{j} \int_{0}^{+\infty} \mathrm{d} R_{k j} \cdot 2 \pi R_{k j} w_{k j}\left(z_{k}, z_{j}, R_{k j}\right)=1 .
$$

Eq. (6) is not useful unless we know the intra-molecular correlation function. Although the second-order distribution function in the bulk is easily obtained [23,32], it is difficult to obtain it for the inhomogeneous system. To proceed, we must make some approximations.

First, we make a Markov-chain approximation for the intra-molecular correlation function. We assume that the correlation function between any two segments can be expressed as:

$$
w_{k j}\left(\mathbf{r}_{k}, \mathbf{r}_{j}\right) \approx C_{k j}^{-1} \int \mathrm{d} \mathbf{r}_{1} \int \mathrm{d} \mathbf{r}_{N} \widetilde{w}_{1 k}\left(\mathbf{r}_{1}, \mathbf{r}_{k}\right) \widetilde{w}_{k j}\left(\mathbf{r}_{k}, \mathbf{r}_{j}\right) \widetilde{w}_{j N}\left(\mathbf{r}_{j}, \mathbf{r}_{N}\right)
$$

where $\widetilde{w}_{i j}$ is the non-normalized correlation function of a chain with chain length $j-i+1$ and $C_{k j}$ is the normalization constant to make Eq. (10) satisfy the normalization condition Eq. (9). $\widetilde{w}_{i j}$ is given by:

$$
\widetilde{w}_{i j}\left(\mathbf{r}_{i}, \mathbf{r}_{j}\right)=\int \cdots \int \prod_{l=i}^{j} \prod_{m=l+2}^{j} \exp \left(-\beta \phi_{l m}\right) \prod_{l=i}^{j} \exp \left(-\beta v_{l}\right) \prod_{l=i}^{j-1} s_{l, l+1} \prod_{l=i+1}^{j-1} \mathrm{~d} \mathbf{r}_{l} .
$$

$C_{k j}$ is given by:

$$
C_{k j}=V^{-1}\left(H_{1}+H_{2}\right) \int \mathrm{d} \mathbf{r}_{1} \int \mathrm{d} \mathbf{r}_{k} \int \mathrm{d} \mathbf{r}_{j} \int \mathrm{d} \mathbf{r}_{N} \widetilde{w}_{1 k}\left(\mathbf{r}_{1}, \mathbf{r}_{k}\right) \widetilde{w}_{k j}\left(\mathbf{r}_{k}, \mathbf{r}_{j}\right) \widetilde{w}_{j N}\left(\mathbf{r}_{j}, \mathbf{r}_{N}\right) .
$$

For a Gaussian-chain or random-flight model, Eq. (10) is exact and the normalization constant $C_{k j}$ is the partition function.

Second, we adopt an assumption for the non-normalized end-to-end correlation function. Considering that the deviation of a real polymer from an ideal polymer arises from the intra-molecular interaction between the segments, the correlation for the real polymer $\widetilde{w}_{i j}$ can be written as: 


$$
\widetilde{w}_{i j}\left(\mathbf{r}_{i}, \mathbf{r}_{j}\right)=\widetilde{w}_{i j}^{\text {id }}\left(\mathbf{r}_{i}, \mathbf{r}_{j}\right) \exp \left(-\beta U_{\text {intra }}\left(\mathbf{r}_{i}, \mathbf{r}_{j} ;[v]\right)\right)
$$

where $\widetilde{w}_{i j}^{\mathrm{id}}$ is the non-normalized correlation function for a Gaussian chain; $U_{\text {intra }}$ is the effective intra-molecular energy of the real chain with segment $i$ at $\mathbf{r}_{i}$ and segment $j$ at $\mathbf{r}_{j}$; and [v] represents the functional dependence on the external potential. We assume that the effective intra-molecular energy of an inhomogeneous system depends weakly on the external field, i.e.

$$
U_{\text {intra }}\left(\mathbf{r}_{i}, \mathbf{r}_{j} ;[v]\right) \approx U_{\text {intra }}\left(\left|\mathbf{r}_{i}-\mathbf{r}_{j}\right|\right) .
$$

Thus, we can use the correlation function in the bulk to estimate the effective intra-molecular energy $U_{\text {intra }}$ of the inhomogeneous system. We obtain:

$$
\widetilde{w}_{i j}\left(\mathbf{r}_{i}, \mathbf{r}_{j}\right) \approx \widetilde{w}_{i j}^{\mathrm{id}}\left(\mathbf{r}_{i}, \mathbf{r}_{j}\right) \exp \left(-\beta U_{\text {intra }}\left(\left|\mathbf{r}_{i}-\mathbf{r}_{j}\right|\right)\right)=\widetilde{w}_{i j}^{\mathrm{id}}\left(\mathbf{r}_{i}, \mathbf{r}_{j}\right) \frac{\widetilde{w}_{i j, 0}\left(\left|\mathbf{r}_{i}-\mathbf{r}_{j}\right|\right)}{\widetilde{w}_{i j, 0}^{\mathrm{id}}\left(\left|\mathbf{r}_{i}-\mathbf{r}_{j}\right|\right)}
$$

where subscript 0 represents a homogeneous system. The normalized correlation function $w_{i j, 0}$ can be found in references [23, 32]. The undetermined normalization constant of the non-normalized correlation function is cancelled with the normalization constant $C_{i j}$ in Eq. (12). The non-normalized correlation function of a Gaussian chain is given by its Green function:

$$
\widetilde{w}_{i j}^{\mathrm{id}}\left(\mathbf{r}_{i}, \mathbf{r}_{j}\right)=G\left(\mathbf{r}_{i}, \mathbf{r}_{j} ; \sigma(j-i)\right)=G^{(z)}\left(z_{i}, z_{j} ; \sigma(j-i)\right) G^{(R)}\left(R_{i j} ; \sigma(j-i)\right)
$$

where superscripts $(z)$ and $(R)$ represent, respectively, the $z$-axis direction and the radial direction and $\sigma(j-i)$ is the chain length of the corresponding Gaussian chain. In the homogeneous system, the Green function is given by a Gaussian distribution function $[2,10]$ :

$$
G\left(\mathbf{r}_{i}, \mathbf{r}_{j} ; \sigma(j-i)\right)=\left(\frac{3}{2 \pi \sigma^{2}(j-i)}\right)^{3 / 2} \exp \left\{-\frac{3\left[\left(x_{i}-x_{j}\right)^{2}+\left(y_{i}-y_{j}\right)^{2}+\left(z_{i}-z_{j}\right)^{2}\right]}{2 \sigma^{2}(j-i)}\right\} .
$$

In the external field, similar to (but not the same because of the different unit), Eq. (1), the Green function of a Gaussian chain in the $z$-axis direction, is given by:

$$
G^{(z)}\left(z_{i}, z_{j} ; \sigma(j-i)\right)=\sum_{k=0}^{+\infty} u_{k}\left(z_{i}\right) u_{k}\left(z_{j}\right) \exp \left[-\beta E_{k} \sigma(j-i)\right]
$$

where $u_{k}(z)$ and $E_{k}$ are, respectively, eigenfunctions and eigenenergies of the second-order ordinary differential equation:

$$
\sigma u^{\prime \prime}(z)-6 \beta v(z) u(z)=-6 \beta E u(z)
$$

with the boundary condition: 


$$
u\left(-H_{1}\right)=u\left(H_{2}\right)=0 .
$$

The analytic expression for Eq. (18) is given in our previous work [31]. Substituting Eq. (16) into Eq. (15), we obtain:

$$
\widetilde{w}_{i j}\left(\mathbf{r}_{i}, \mathbf{r}_{j}\right)=\widetilde{w}_{i j, 0}\left(\left|\mathbf{r}_{i}-\mathbf{r}_{j}\right|\right) G^{(z)}\left(z_{i}, z_{j} ; \sigma(j-i)\right) \sqrt{\frac{2 \pi \sigma^{2}(j-i)}{3}} \exp \left(\frac{3\left(z_{i}-z_{j}\right)^{2}}{2 \sigma^{2}(j-i)}\right) .
$$

For the nearest-neighbor pair, we need not use Eq. (21); we know it exactly. The non-normalized correlation function of a dimer is given by:

$$
\widetilde{w}_{k-1, k}\left(\mathbf{r}_{k-1}, \mathbf{r}_{k}\right)=\exp \left\{\left[-\beta v\left(z_{k-1}\right)-\beta v\left(z_{k}\right)\right] / 2\right\} s_{k-1, k}\left(\left|\mathbf{r}_{k-1}-\mathbf{r}_{k}\right|\right) .
$$

The factor 2 comes from our definition of the external potential, Eq. (1), because for a dimer, both segments $k$ and $k-1$ are end segments.

With the help of two approximations, Eqs. (10) and (15), Eq. (6) is now ready for integration. The solution of Eq. (6) is given by:

$$
\begin{aligned}
& \rho_{k}(z)=\exp \left(-\beta v_{k}(z)\right)\left[C+\int_{-H_{1}}^{z} f(t) \exp \left(\beta v_{k}(z)\right) \mathrm{d} t\right] \\
& C=\left[1-\int_{-H_{1}}^{H_{2}} \mathrm{~d} z \exp \left(-\beta v_{k}(z)\right) \int_{-H_{1}}^{z} \mathrm{~d} t f(t) \exp \left(\beta v_{k}(z)\right)\right] / \int_{-H_{1}}^{H_{2}} \mathrm{~d} z \exp \left(-\beta v_{k}(z)\right)
\end{aligned}
$$

where $f(z)$ stands for the last three terms on the right-hand side of Eq. (6). The density distribution of the segments is given by:

$$
\rho(z)=\frac{1}{N} \sum_{k=1}^{N} \rho_{k}(z) .
$$

Eq. (15) can be used to obtain the structure information of the polymer. Similar to the end-to-end-distance distribution function in the bulk, we have the averaged end-to-end-distance distribution function in the external potential. The average is taken over the whole space. The averaged end-to-end-distance distribution function is given by:

$$
\left\langle w_{1 N}(r)\right\rangle=2 \pi \int_{-H_{1}}^{H_{2}} \mathrm{~d} z_{1} \int_{-H_{1}}^{H_{2}} \mathrm{~d} z_{N} \int_{0}^{+\infty} \mathrm{d} R \cdot \delta\left(r-\sqrt{\left(z_{1}-z_{N}\right)^{2}+R^{2}}\right) R w_{1 N}\left(z_{1}, z_{N}, R\right)
$$

where $w_{1 N}\left(z_{1}, z_{N}, R\right)$ is calculated from Eq. (15). The average end-to-end-distance distribution function in z-axis direction is given by:

$$
\left\langle w_{1 N}^{(z)}(z)\right\rangle=2 \pi \int_{0}^{+\infty} \mathrm{d} R \cdot R\left\langle w_{1 N}\left(\sqrt{z^{2}+R^{2}}\right)\right\rangle .
$$




\section{MONTE CARLO SIMULATION}

We consider hard-sphere chains and square-well chains. The segment-segment interaction energy is given by:

$$
\beta \phi(r)=\left\{\begin{array}{c}
+\infty ; \quad r<\sigma \\
\beta \zeta ; \quad \sigma<r<\lambda \sigma \\
0 ; \quad r>\lambda \sigma
\end{array}\right.
$$

where $\zeta$ is the well depth and $\lambda$ is a constant between 1 and 2 . When $\zeta$ or $\lambda$ vanishes, Eq. (27) degenerates to the hard-sphere potential. The appendix of reference [32] gives the derivative of $\beta \phi$ with respect to $r$ and some useful intermediate quantity such as the value of the correlation function at the discontinuous point of the potential $\phi$.

We use the Dickman-Hall algorithm [33] for the canonical Monte-Carlo simulations. The procedure for simulation is as follows. First, the initial configuration of the copolymer is generated by an off-lattice self-avoiding random walk. Second, the chain is subjected to a "translate-jiggle" movement. A bond is chosen randomly on the chain. Then, we randomly choose either one end (e.g. segment $i$ ) of the bond to be stationary and make the other end of the bond (segment $i-1$ ) translate a certain distance. Then, we normalize the distance between these two segments to be the bond length, i.e. normalize $r_{i-1 . i}$ to $\sigma$. The sub-chain (segments $1,2 \ldots i-2$ ) that is connected to segment $i-1$ is subjected to the same translation as that for segment $i-1$. The Metropolis algorithm is used to decide if the movement is accepted or not. Then, a procedure similar to moving segment $i-1$ is applied repeatedly to segment $i-2$ and so on until the end of the chain.

\section{RESULTS AND DISCUSSION}

We use Eq. (23) to investigate how the potential well and segment-segment interactions affect adsorption of the polymer at the interface. The diameter of the segment $\sigma$ is chosen to be a length unit, i.e. $\sigma=1$. The well is located at the center of the box in the $z$-axis direction such that the center of the well is at $z=0$. We discretize the $z$-axis into small boxes by step size $\Delta z=0.1 \sigma$. The simple trapezoidal rule [34] is used for all integrations. Eq. (15) involves a small positive number $\left(G_{i j, 0}\right)$ dividing a positive number $\left(w_{i j, 0}\right)$. Since $w_{i j, 0}$ is obtained numerically, we set $w_{i j, 0}=0$ if its value is smaller than $10^{-12}$. The width of the system is set to be a large number $200 \sigma$ where $H_{1}=H_{2}=100 \sigma$. The well depth $\zeta$ of the segment-segment interaction depends on the solvent. 
We do not include the dependence of $\zeta$ on solvent into the theory. Thus the square-well chain is considered in the symmetric external potential only.

Figs. 1a and $1 \mathrm{~b}$ show how the density distribution of hard chains depends on the chain length. The well depth and well width are, respectively, $\varepsilon_{0}=\varepsilon_{1}=0.5$ and $a=\sigma$. Adsorption rises with increasing chain length. When the chain length is larger than a certain value, the density distribution is close to the limiting case of an infinitely long chain. For a shorter chain, the theory is in good agreement with Monte Carlo simulations. For a longer chain, the theory predicts adsorption weaker than that from simulation, due to our second approximation. In that approximation, Eq. (15), we use the effective intra-molecular energy in the bulk to estimate that in the external field. For a shorter chain, the adsorption is not strong. Because the configuration of the polymer does not change much, the approximation is a good one.

For a longer chain, however, adsorption is very strong. The configuration of the polymer changes from a sphere to a flat-plate-like ellipsoid. Our second approximation is insufficient to estimate the variation of the configurational change; thus, the predicted adsorption is weaker than that from simulation. To see this clearly, we show in Figs. $2 a$ and 2b, respectively, average end-to-end distribution functions and their components in the $z$ direction for a 16-mer, 32-mer and 64-mer. Lines are calculated from Eq. (25); dots are from Monte Carlo simulations. The simulation data show that although the chain span averaged over the whole space of a 64-mer is much larger than that of a 16 mer or a 32-mer as shown in Fig. 2a, the chain span in the $\mathrm{z}$ direction of the 64-mer is similar to those of the 16-mer and the 32-mer as shown in Fig. 2b. A longer chain is more likely to be in a flat-plate configuration in the interface than a shorter chain. The deviation of Eq. (15) for the shorter chain is smaller than that for the longer chain. Therefore, the results of the theory are better for short chains. It follows from the discussion above, if the well depth of the external field is small, the theory can be used for a longer chain. 


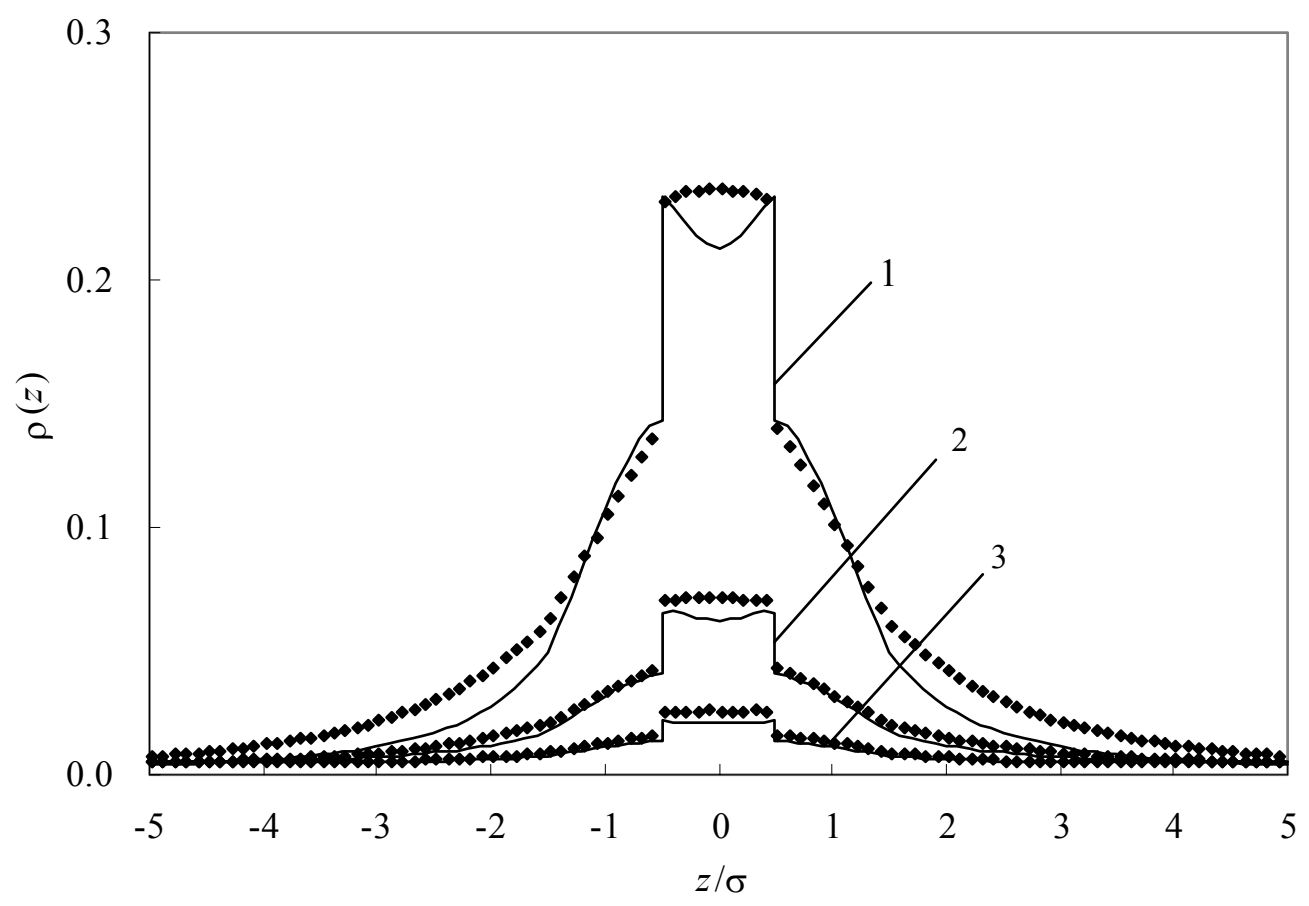

Fig. 1a Density distributions for hard chains in a symmetric square well with well width $a=\sigma$ and well depth $\beta \varepsilon_{0}=\beta \varepsilon_{1}=0.5$. 1. 32-mer; 2. 16-mer; 3. 8-mer. Lines: theory; Dots: simulations

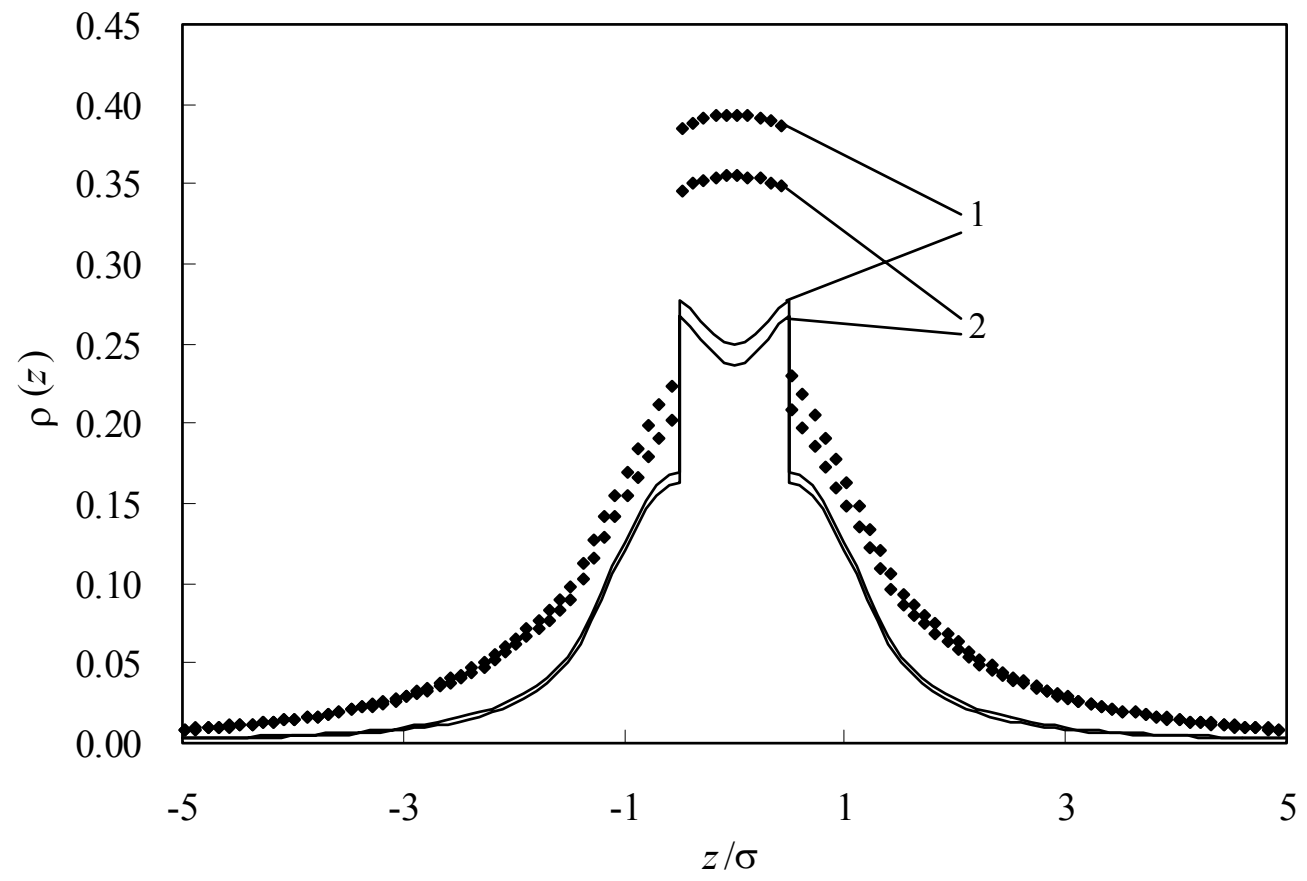

Fig. 1b Density distributions for hard chains in a symmetric square well as in Fig. 1a.

1. 64-mer; 2. 48-mer. Lines: theory; Dots: simulations 


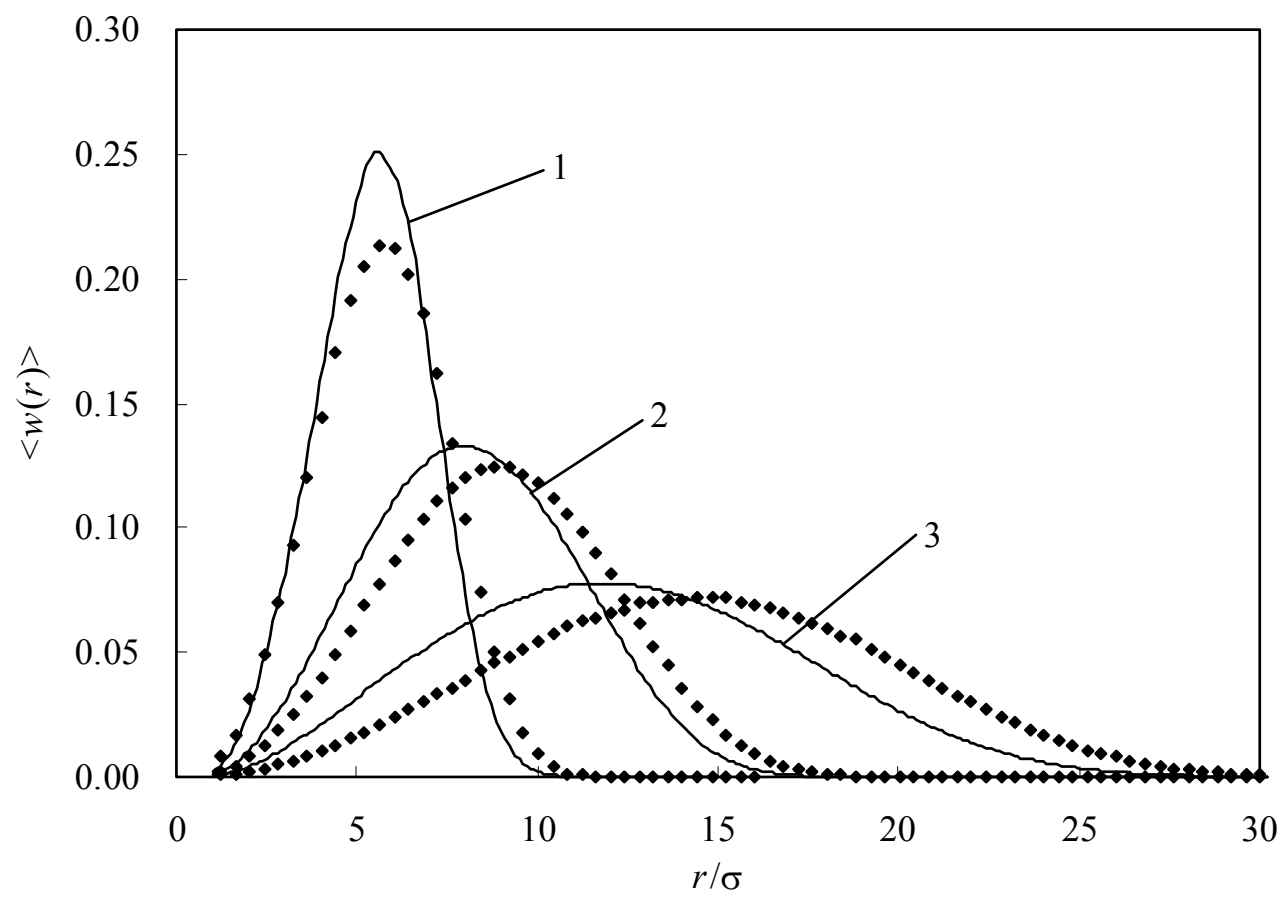

Fig. 2a The average end-to-end-distance distribution function in a symmetric square well as in Fig.

1a. 1. 16-mer; 2. 32-mer; 3. 64-mer. Lines: theory; dots: simulations.

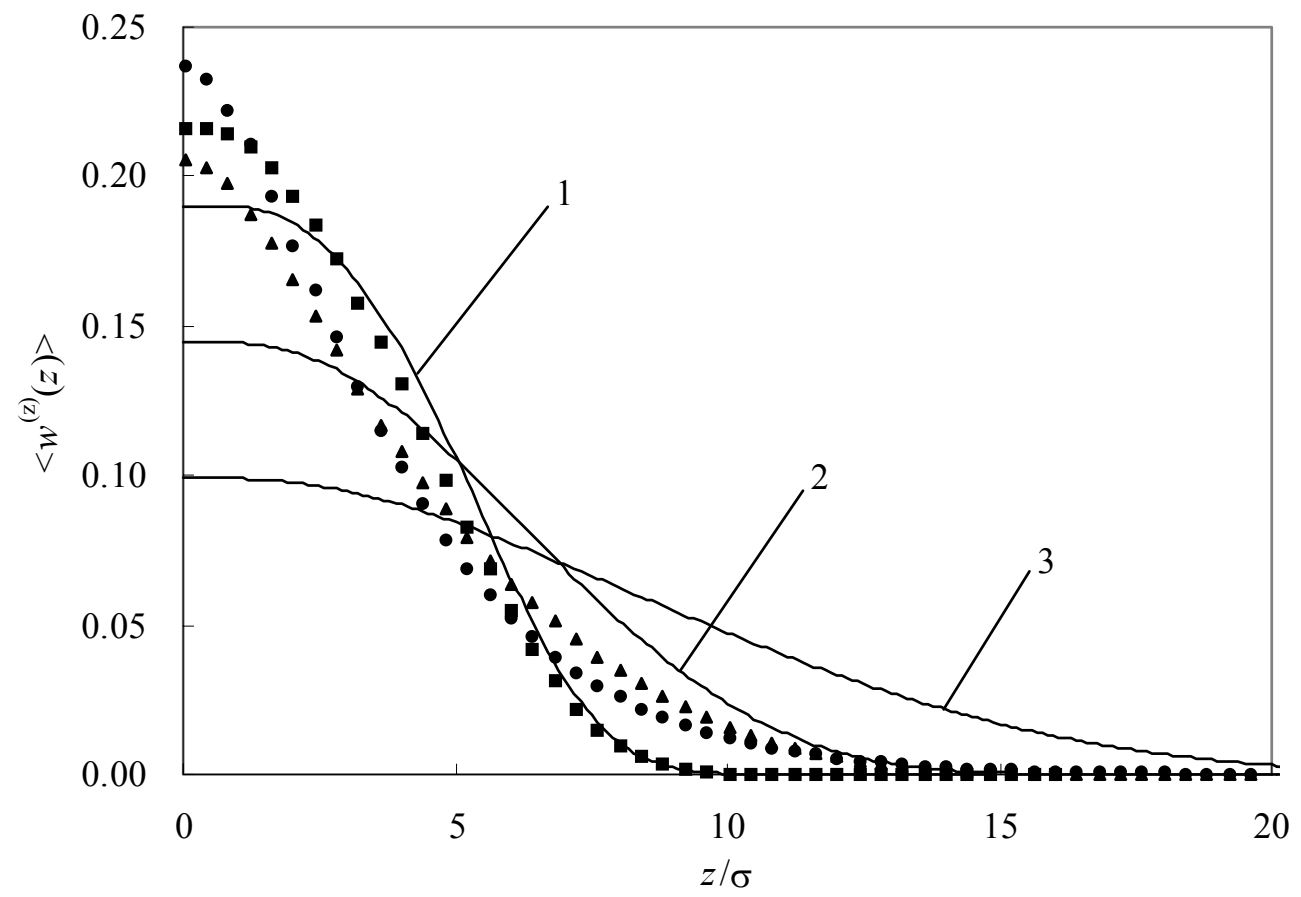

Fig. $2 \mathrm{~b}$ The average end-to-end-distance distribution function in $\mathrm{z}$ for a symmetric square well as in Fig. 1a. 1. 16-mer; 2. 32-mer; 3. 64-mer. Lines: theory; dots: simulations. 
Fig. 3 shows how attractive interaction influences adsorption of a 32-mer. Fig. 4 shows the average end-to-end-distance distribution function and its component in the $z$ direction. The external potential is the same as that in Fig. 1a. The well depth $\beta \zeta$ of the interaction between segments is taken as 0 and -0.3 . When the attractive interaction becomes stronger, adsorption increases. When there are attractive interactions, we have competition between the configurational entropy and the energy due to the interface. On the one hand, the attractive interaction makes the polymer more compact such that more segments can be at the interface. On the other hand, the attractive interaction tends to keep the polymer in a sphere to make more contacts between segments. When the attractive interaction is not very strong, the energy effects due to the interface dominate. Thus, with increasing attraction, adsorption increases. As shown in reference [23], for a square-well chain in the bulk, the scaling law (radius of gyration: $\left\langle R_{g}^{2}\right\rangle \sim N^{2 \gamma}$ ) shows that the well depth $\beta \zeta=-0.3$ corresponds to the theta chain. However, due to interactions between segments, the density distribution from a random-flight model is different from that of a square-well chain with $\beta \zeta=-0.3$. Adsorption from a random-flight model is much stronger than that for a "theta" square-well chain. To investigate how the external field influences the scaling law of the polymer, we need to calculate the structure property of a very long chain. Our theory is better for a short chain. 


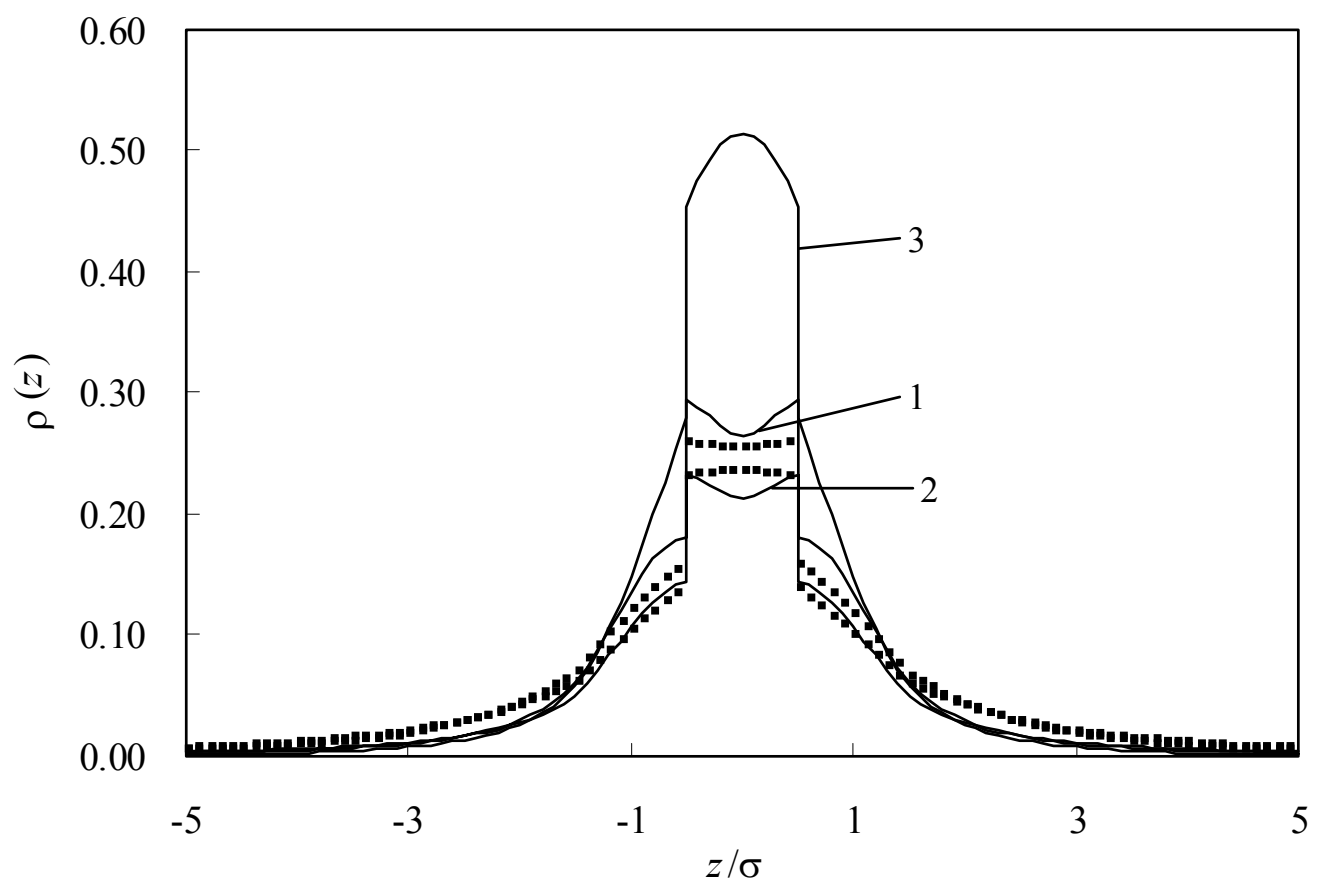

Fig.3 Density distributions for a hard 32-mer and a square-well 32-mer in a symmetric square well as in Fig. 1a. $1 . \beta \zeta=-0.3 ; 2 . \beta \zeta=0 ; 3$. Random flight model. Lines: theory; dots: simulations.

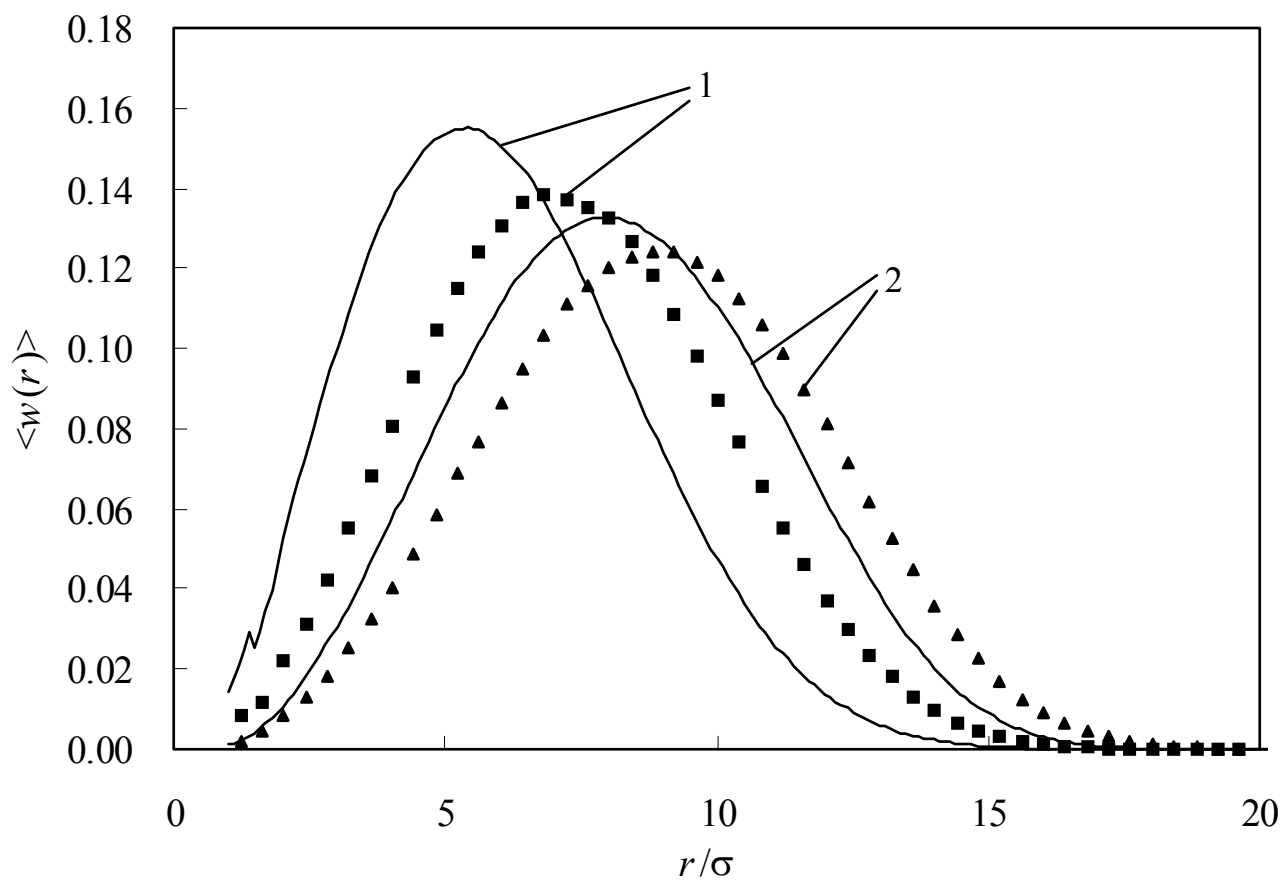

Fig. 4a The average end-to-end-distance distribution function in a symmetric square well as in Fig. 1a. 1. $\beta \zeta=-0.3 ; 2$. $\beta \zeta=0$. Lines: theory; dots: simulations. 


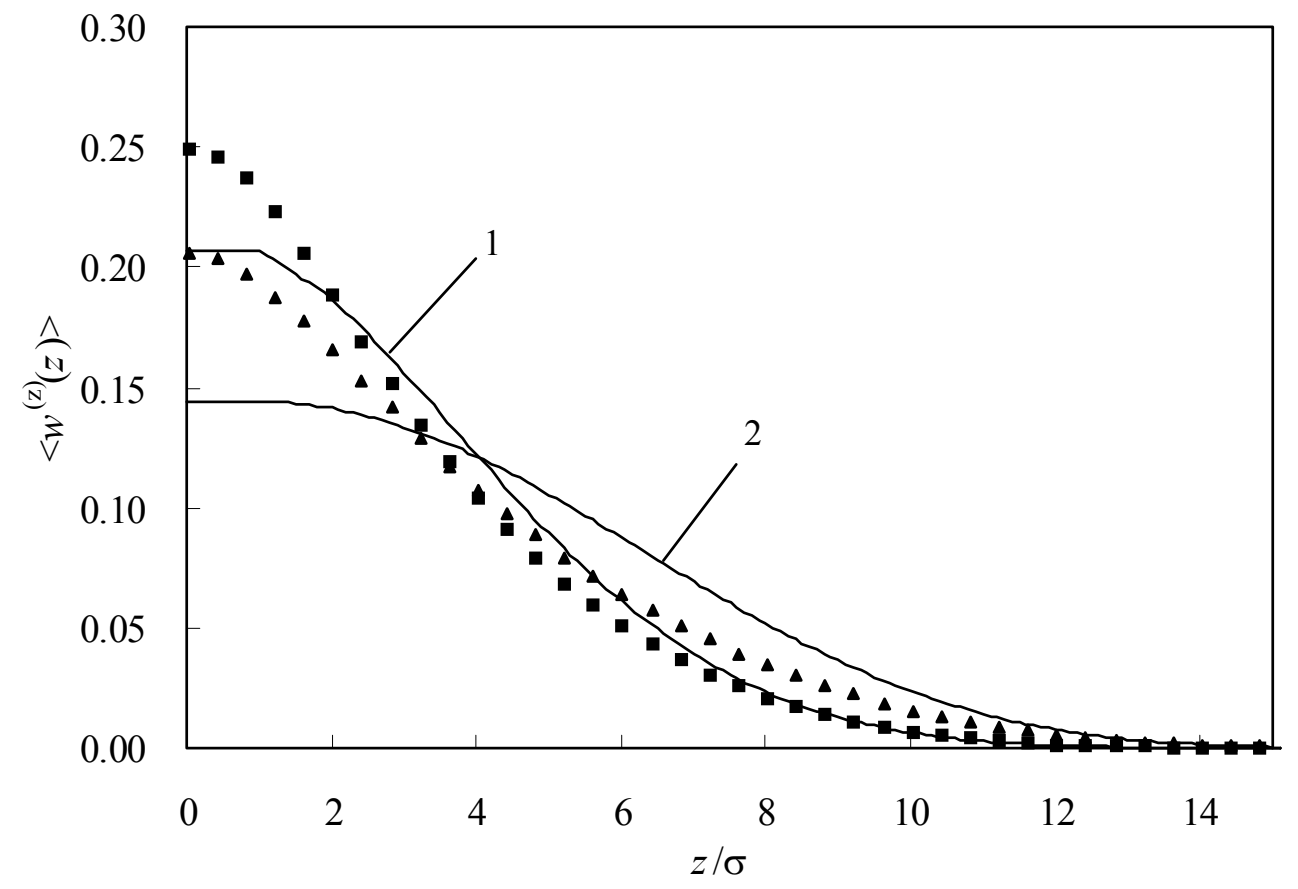

Fig. $4 \mathrm{~b}$ The average end-to-end-distance distribution function in the $\mathrm{z}$ direction for a symmetric square well as in Fig. 1a. $1 . \beta \zeta=-0.3 ; 2 . \beta \zeta=0$. Lines: theory; dots: simulations.

Figs. 3 and 4 are for the square-well chain with relatively weak attractive interactions; adsorption of the polymer rises with increasing attraction. For very strong attractive interaction, the theory predicts a very large depletion layer inside the interface. This result follows from our second approximation in Eq. (15). References [23] and [32] show that the BGY equation overestimates the collapse. The stronger the collapse, the stronger the depletion layer. We must rely on the Monte Carlo simulation to determine how strong attractive interactions influence adsorption.

Fig. 5 shows the density distribution function for a 64-mer with various attractive interactions. The configurational-entropy effect causes a depletion layer inside the interface for very strong attractions between segments.

Fig. 6 shows how attractive interactions influence adsorption. To describe how many segments are near the interface, we define a quantity $W$ that is the probability to find the polymer near the interface at a region that is about $10 \sigma$ wide. $W$ is given by:

$$
W=\int_{-5 \sigma}^{5 \sigma} \mathrm{d} z \cdot \rho(z) .
$$


When the attraction is very strong, adsorption tends to a limit. For much stronger interaction, the polymer becomes a glass [23], beyond the scope of this work.

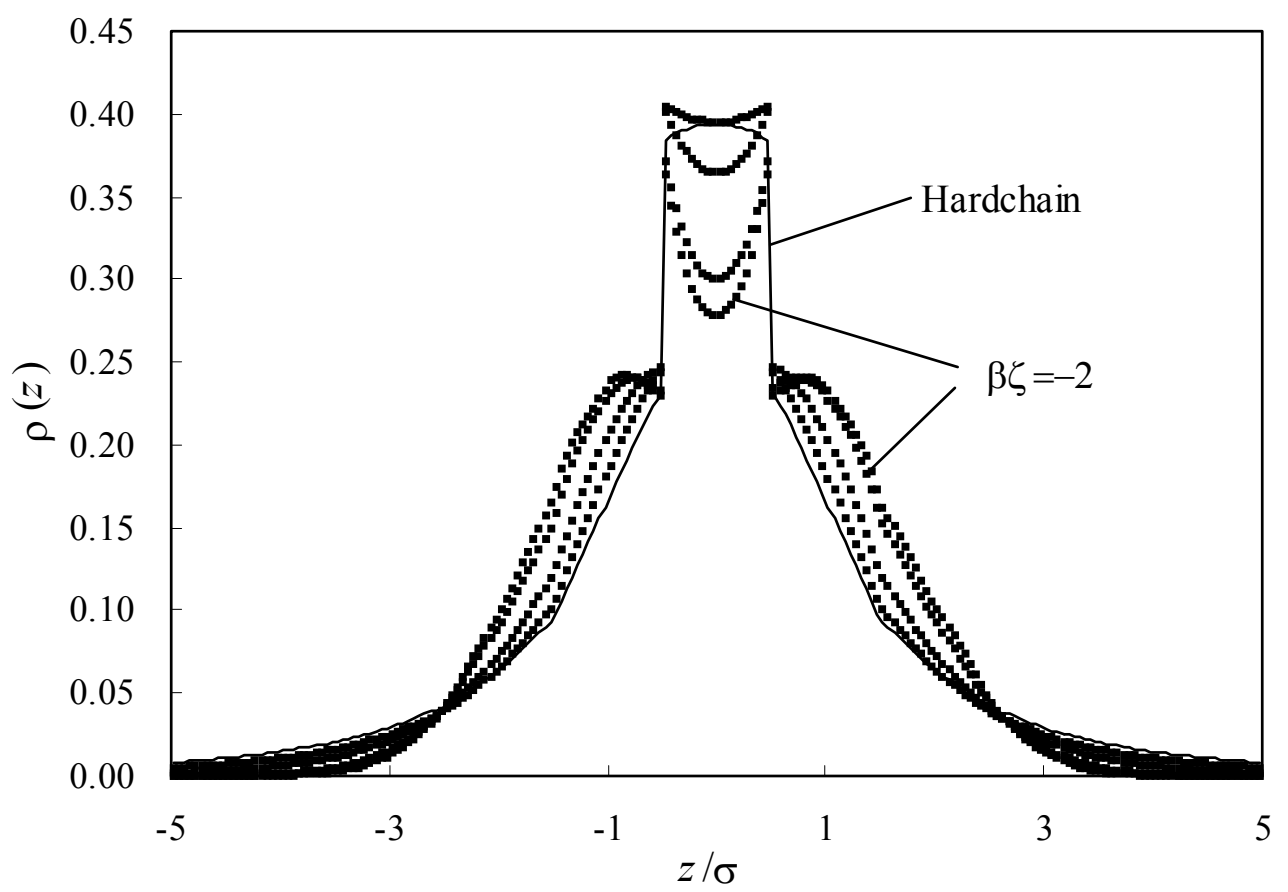

Fig.5 Density distributions for square-well 64-mer in a symmetric square well as in Fig. 1a. Line: $\beta \zeta=0$; dots: $\beta \zeta=-0.3,-0.5,-1,-2$

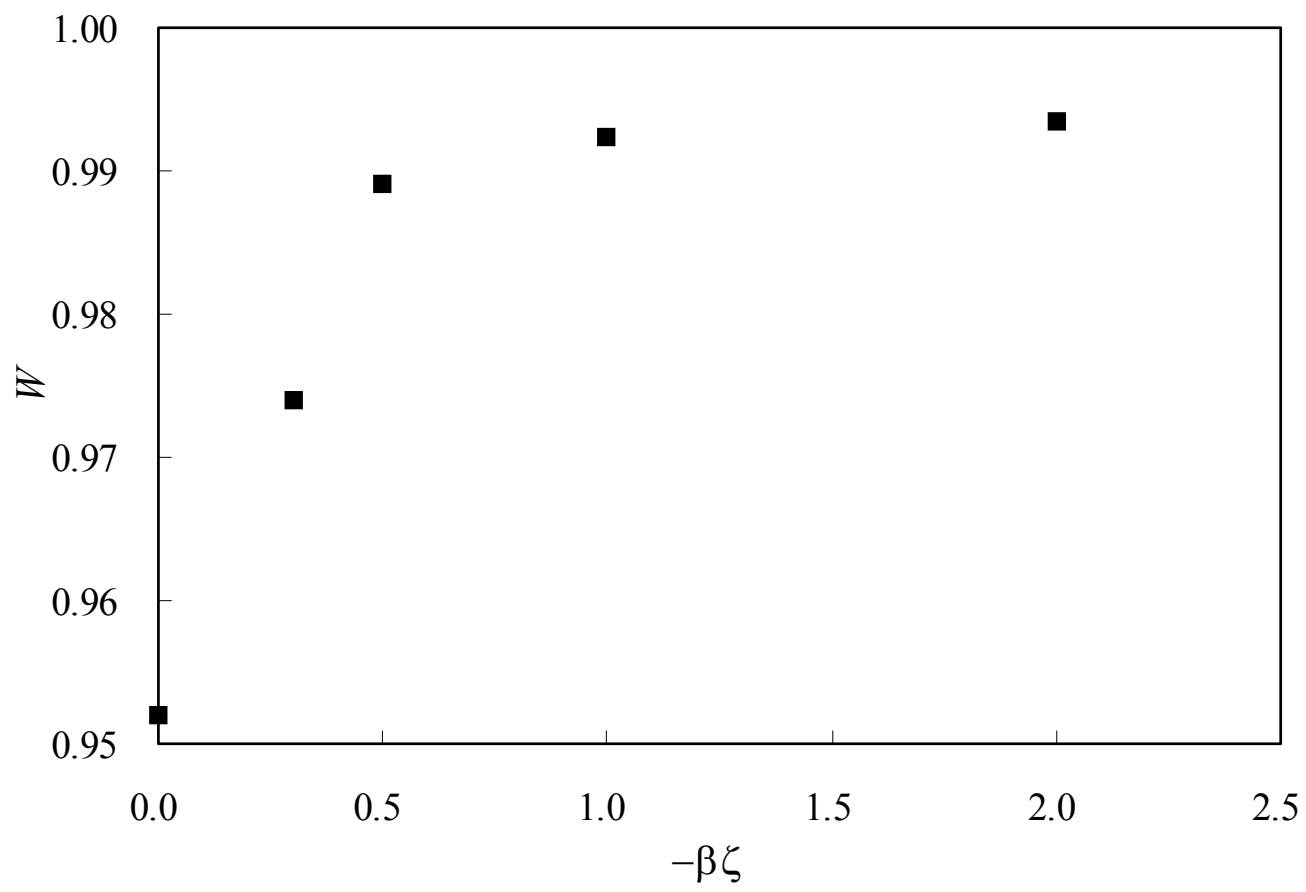

Fig. 6 The probability to find the polymer near a symmetric square well as in Fig. 1a. 


\section{CONCLUSIONS}

First, to describe adsorption of a self-interacting polymer at the interface, we have established an integral-equation theory based on the BGY hierarchy. The two approximations that we introduced to truncate the BGY hierarchy represent the structure of a hard chain or a square-well chain adsorbed at the interface. For a short chain, the theory is in good agreement with Monte Carlo simulations. For a longer chain, our approximations do not take into account the long-range correlation due to the connection between segments. Deviation of the theory mainly arises from the second approximation where we use the effective intra-molecular energy of a polymer in the bulk to approximate that at the interface. When the chain is long, the conformation of the polymer at the interface is significantly different from that in the bulk and the effective intra-molecular energy changes too much. Thus, our theory is better for a short chain than for a long chain.

Second, to take into account the attractive interaction beyond volumetric repulsion, the widely used field theory and renormalization-group (RG) theory are not suitable. In our previous work [22], we derived a differential equation for a polymer adsorbed at an interface based on the standard field theory. We found that there exists a physically meaningless bound state even when the external field vanishes. (This bound state has been used to explain the collapse of the polymer in the bulk.) This implies that the bound state is an artifact that arises from the ill-defined equation itself and does not correspond to the real collapse of the polymer. This problem probably arises from the Dirac-function-like interaction between segments. However, for a more realistic potential, the field theory becomes impractical. RG theory is successful in the self-avoiding-walk problem. Because it is based on the field theory, RG is impractical when there is attractive interaction between segments. Thus, it seems that we have to use the integral-equation theory for attractive interactions. Regrettably, however, for long chains our second approximation does not correctly take into account the long-range correlation.

Third, our numerical results show that, similar to results for a Gaussian chain, when we have adsorption, a longer chain length favors adsorption due to the connections between segments and due to the energy decrease at the interface. The configuration of a polymer chain is similar to that of a plate parallel to the surface. Adsorption of a polymer with a weak to moderate attractive interaction between segments is stronger than that with repulsion only. When we have attractive interactions, there is a competition between the configurational entropy and the energy due to the surface. When the attractive interaction is not very strong, the energy effects due to the interface 
dominate, i.e. the attractive interaction favors adsorption. For the polymer with strong attractive interaction, our theory is not useful. However, the Monte Carlo simulations show that with increasing attractive interaction, adsorption increases and gradually approaches a limit.

\section{Acknowledgement}

For financial support, the authors are grateful to the Office for Basic Sciences, US Department of Energy and to the Chinese National Science Foundation.

\section{Literature Cited:}

1. See P. G. de Gennes, Macromolecules, 14, 1637 (1981) and references cited there.

2. P. G. de Gennes, Scaling Concepts in Polymer Physics, Cornell University Press, Ithaca and London, 1979.

3. E. Eisenriegler, Polymers Near Surfaces, World Scientific, Singapore, 1993.

4. M. Tupy, H. Blanch, C. Radke, Ind. Eng. Chem. Res., 37, 3159 (2001).

5. C. Beverung, C. Radke, H. Blanch, Biophys. Chem., 81, 59 (1999).

6. R. Anderson, V. Pande, C. Radke, J. Chem. Phys., 112, 9167 (2000).

7. Z-G. Wang, A. Nemirovsky, K. Freed, J. Chem. Phys., 85, 3068 (1986).

8. S. Stepanow, U. Bauerschafer, J. Sommer, Phys. Rev. E54, 3899 (1996).

9. G. Stratouras, M. Kosmas, J. Chem. Phys. 95, 4656 (1991).

10. K. Freed, Renormalization Group Theory of Macromolecules, John Wiley and Sons, New York, 1987.

11. S. Stepanow, J. Chem. Phys., 115, 1565 (2001).

12. I. Teraoka, P. Cifra, J. Chem. Phys., 115, 11362 (2001).

13. S. Edwards, Proc. Phys. Soc. (London), 85, 613 (1965).

14. K. Freed, Adv. Chem. Phys., 22, 1 (1972).

15. K. Freed, H. Gillis, Chem. Phys. Lett., 8, 384 (1971).

16. M. Kosmas, K. F. Freed, J. Chem. Phys., 68, 4878 (1978).

17. H. Yamakawa, Modern Theory of Polymer Solutions, Harper \& Row, New York, 1971.

18. A. Kholodenko, K. Freed, J. Phys. A: Math. Gen., 17, 2703 (1984).

19. J. des Cloizeaux, J. Phys. Lett. (Paris), 41, L151 (1980); J. Phys. (Paris), 41, 223 (1980).

20. Y. Oono, K. Freed, J. Chem. Phys., 75, 993, 1009 (1981).

21. Y. Oono, Adv. Chem. Phys., 61, 301 (1985). 
22. J. Cai, J. Prausnitz, submitted to J. Chem. Phys.

23. M. P. Taylor and J. E. G. Lipson, J. Chem. Phys. 104, 4835 (1996).

24. M. P. Taylor, Mol. Phys. 86, 73 (1980).

25. B. C. Eu and H. H. Gan, J. Chem. Phys. 99, 4804 (1993).

26. H. H. Gan and B. C. Eu, J. Chem. Phys. 99, 4103 (1993), 110, 3235 (1999).

27. T. Hill, Statistical Mechanics, Dover, New York, 1956.

28. J. Hansen and I. McDonald, Theory of Simple Liquids, $2^{\text {nd }}$ ed., Academic Press, 1987.

29. M. Born and H. Green, Proc. R. Soc. London, Ser. A 188, 10 (1946).

30. S. Whittington and L. Dunfield, J. Phys. A6, 484 (1973).

31. J. Cai and J. Prausnitz, J. Chem. Phys. 117, 3935 (2002).

32. J. Cai and J. Prausnitz, submitted to J. Chem. Phys.

33. R. Dickman and C. Hall, J. Chem. Phys. 85, 4108 (1986).

34. W. Press, S. Teukolsky, W. Vetterling and B. Flannery, Numerical Recipes in C, the Art of Scientific Computing, $2^{\text {nd }}$ edition, Cambridge University Press, Cambridge, 1992.

35. Y. Zhou, C. Hall and M. Karplus, Phys. Rev. Lett. 77, 2822 (1996); Y. Zhou, M. Karplus, J. Wichert and C. Hall, J. Chem. Phys. 107, 10691 (1997). 